\title{
A RARE CASE OF DEDIFFERENTIATED LIPOSARCOMA WITH CHONDROSARCOMATOUS DEDIFFERENTIATION
}

Tanu Agrawal ${ }^{1}$, Kanchan Garg², Dupinder Kaur 3 , S. K. Sagar ${ }^{4}$

\section{HOW TO CITE THIS ARTICLE:}

Tanu Agrawal, Kanchan Garg, Dupinder Kaur, S. K. Sagar. "A Rare Case of Dedifferentiated Liposarcoma with Chondrosarcomatous Dedifferentiation". Journal of Evolution of Medical and Dental Sciences 2015; Vol. 4, Issue 48, June 15; Page: 8425-8429, DOI: 10.14260/jemds/2015/1221

\begin{abstract}
We report a rare case of dedifferentiated liposarcoma with chondrosarcomatous components. A 39 years old male presenting with a left buttock swelling underwent a wide tumor excision. The histopathological diagnosis was dedifferentiated liposarcoma (DDLS) with chondrosarcomatous dedifferentiation. Histologically, DDLS is represented by the abrupt transition from well differentiated liposarcoma to a region of non-lipogenic sarcoma. Dedifferentiation frequently resembles a malignant fibrous histiocytoma-like pleomorphic sarcoma. Divergent differentiation with chondrosarcomatous components is very rare. We present here a case of gluteal dedifferentiated liposarcoma with chondrosarcomatous dedifferentiation because of its extreme rarity.
\end{abstract}

KEYWORDS: Dedifferentiated liposarcoma, chondrosarcoma, gluteal.

INTRODUCTION: Liposarcomas are the most frequent soft tissue sarcoma in adults. Liposarcomas are usually large and occur most frequently in the lower extremities; retroperitoneal, perirenal, and mesenteric region; and shoulder area. ${ }^{1}$ Included in the general category of liposarcoma is a number of subtypes that are histologically, biologically, cytogenetically and by molecular analyses distinct from one another. According to World Health Organization, it is classified into four subtypes: Atypical Lipomatous Neoplasm/ Well Differentiated Liposarcoma, Myxoid/Round cell, Dedifferentiated and Pleomorphic liposarcoma. ${ }^{2}$ Dedifferentiated, round cell and pleomorphic liposarcoma are high-grade, aggressive tumors with metastatic potential while well differentiated and myxoid liposarcoma are low-grade tumors that follow a more indolent clinical course. ${ }^{3}$ DDLS has 2 distinct components, a well-differentiated lipomatous component and a dedifferentiated non-lipomatous component composed of sarcomas, such as myxofibrosarcomas or other spindle-cell sarcomas. Dedifferentiated component exhibiting chondrosarcomatous dedifferentiation is very rare with only few cases reported in literature. ${ }^{4,5}$

CASE REPORT: A 49 years old male presented with complaint of swelling in the left buttock. He had noted the swelling approximately 6 months earlier but in last fifteen days before presentation it had rapidly increased in size. It was associated with mild intermittent pain. He denied any associated trauma.

Physical examination revealed a firm, non-tender and non- mobile mass measuring 10x8 cm with normal overlying integument in the left gluteal region. Complete blood cell count and routine biochemical investigations were within normal limits. Fine needle aspiration cytology was done which was inconclusive. Magnetic resonance imaging revealed a large heterogeneous mass in the left gluteal muscle with interspersed cystic and necrotic areas suggestive of soft tissue sarcoma. Patient underwent wide excision of left gluteal region and the resected specimen was sent for histopathological analysis. 


\section{CASE REPORT}

Gross examination revealed a globular mass, measuring $14 \times 10 \times 6 \mathrm{~cm}$ with attached skin flap on one surface. Cut surface was lobulated greyish white to yellow with translucent cartilaginous areas (Figure 1).

Microscopic examination revealed a tumor with lobular areas composed of mature fat cells with interspersed uni- and multivacuolated lipoblasts (Figure 2, 3). In other areas the tumor displayed lipoblasts and anastomosing capillaries in a background of abundant myxoid matrix. Large areas of chondroid differentiation with increased cellularity and binucleated chondrocytes with atypical pleomorphic hyperchromatic nuclei suggestive of chondrosarcoma were also present (Figure 4, 5).

On immunohistochemistry, the tumour cells were positive for S-100 (Figure 6).

On the basis of these findings a diagnosis of dedifferentiated liposarcoma with chondosarcomatous dedifferentiation was made.

Postoperative radiation was offered as adjuvant therapy to the patient but was refused by the family and the patient did not return for follow up.

DISCUSSION: Dedifferentiated liposarcoma (DDLS) occurs in upto 10\% of well differentiated liposarcomas with predilection for retroperitoneum. The phenomenon of dedifferentiation was first described by Dahlin and Beabout. ${ }^{6}$ In 1979, Evans ${ }^{7}$ reassessed that definition and coined the term dedifferentiated liposarcoma, which presents not as a complication but as a primary, de novo, bimorphic neoplasm with components of both a low grade, well differentiated liposarcoma and an entirely histologically different high grade sarcoma. ${ }^{4}$ Morphology of DDLS typically ranges from low to high grade components, commonly resembling myxofibrosarcoma, malignant fibrous histiocytoma or sarcoma not otherwise specified.In contrast, divergent differentiation, including the development of rhabdomyosarcomatous, leiomyosarcomatous, or osteosarcomatous components, has been reported in less than $5 \%$ DDL. $^{8}$ Chondrosarcomatous dedifferentiation is even rarer. DDLS shows high local recurrence and often gives rise to widespread metastasis. The presence of a dedifferentiated component confers aggressive biological behavior with a local recurrence rate of $41 \%$, a metastatic rate of $17 \%$ and disease-related mortality of $28 \% .{ }^{9}$ Surgical excision is the treatment of choice with the use of chemotherapy in metastatic disease. ${ }^{5}$ Adjuvant radiation therapy is selectively utilized in patients with extremity DDLS. ${ }^{3}$

CONCLUSION: We have presented a rare case of dedifferentiated liposarcoma with chondrosarcomatous dedifferentiation. This case highlights the range of heterologous elements that can be seen in DDLS.

\section{REFERENCES:}

1. Juan R. Soft Tissues. In: Juan R, editor. Rosai and Ackerman's Surgical Pathology. 10 $10^{\text {th }}$ edition. Volume 2. Elsevier, 2011. 2145-2146.

2. Fletcher C.D.M, Unni K.K, Mertens F. Pathology and genetics of tumors of soft tissue and bone. Lyon: IARC Press, 2002, 35-46.

3. Crago A.M, Singer S. Clinical and Molecular Approaches to Well-differentiated and Dedifferentiated Liposarcoma. Current Opinion in Oncology. 2011; 23(4): 373-378.

4. Yoon R.S, Benevenia J, Beebe K.S, Hameed M. Dedifferentiated liposarcoma of thigh with chondrosarcomatous dedifferentiated component. Am J Orthop. 2010; 39(11): E114-8. 


\section{CASE REPORT}

5. Patne Shashikant C.U, Kumar M, Vishwanath A, Pandey M. Primary subcutaneous mixed-type liposarcoma of the thigh showing three simultaneous dedifferentiations: Report of an unusual case. Indian Journal of pathology and Microbiology. 2013; 56(4) 419-421.

6. Dahlin D.C, Beabout J.W. Dedifferentiation of low grade chondrosarcomas. Cancer. 1971; 28(2): 461-466.

7. Evans H.L. Liposarcoma: a study of 55 cases with a reassessment of its classification. Am J Surg Pathol. 1979; 3(6): 507-523.

8. Fujii T, Arai T, Sakon $M$, et al. Retroperitoneal dedifferentiated liposarcoma with osteosarcomatous components: a case report. International Journal of Clinical and Experimental Pathology. 2013; 6(7): 1427-1431.

9. Henricks W.H, Chu Y.C, Goldblum J.R, Weiss S.W. Dedifferentiated liposarcoma. A clinicopathological analysis of 155 cases with a proposal for an expanded definition of dedifferentiation. Am J Surg Pathol 1997; 21: 271-81.

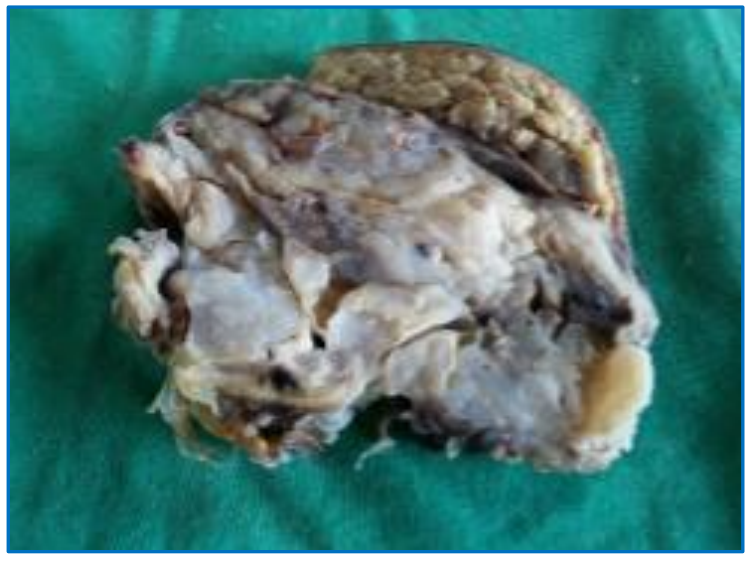

Fig. 1: Gross photograph showing lobulated tumor Mass with yellow fatty and translucent cartilaginous areas

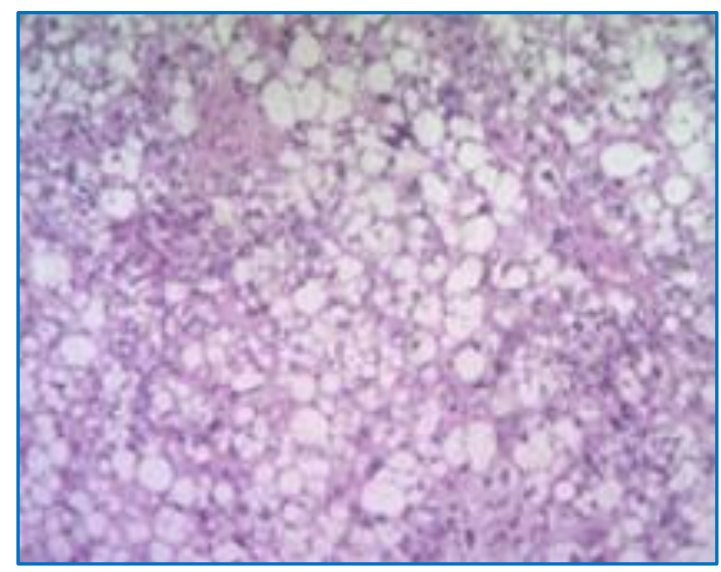

Fig. 2: Microphotograph showing liposarcomatous areas with mature fat and interspersed lipoblasts (H\&E 10X) 


\section{CASE REPORT}

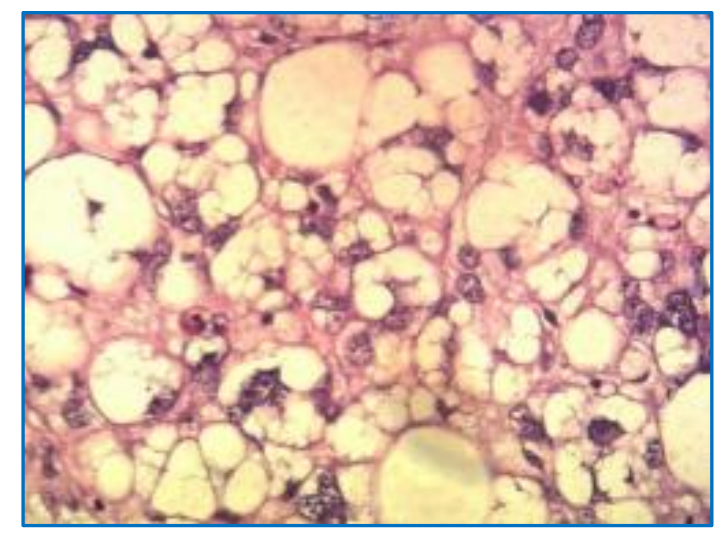

\section{Fig. 3: Microphotograph showing many} multivacuolated lipoblasts (H\&E 40X)

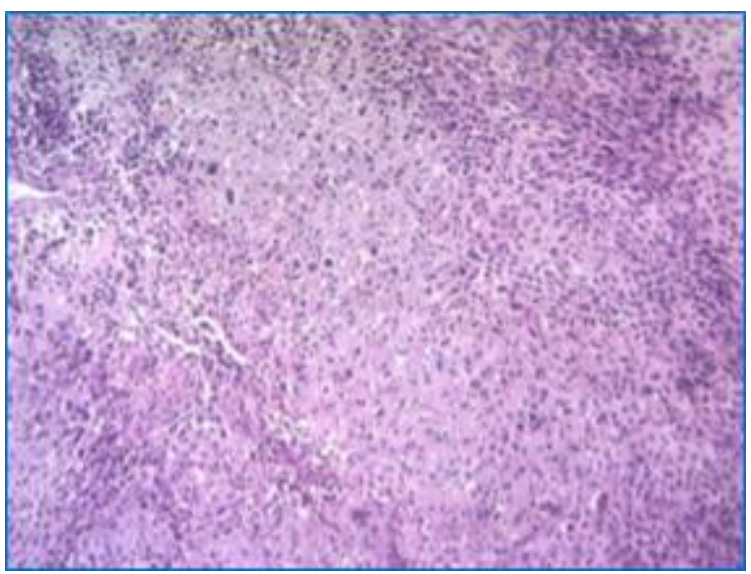

Fig. 4: Microphotograph showing chondrosarcomatous area (H\&E 10X)

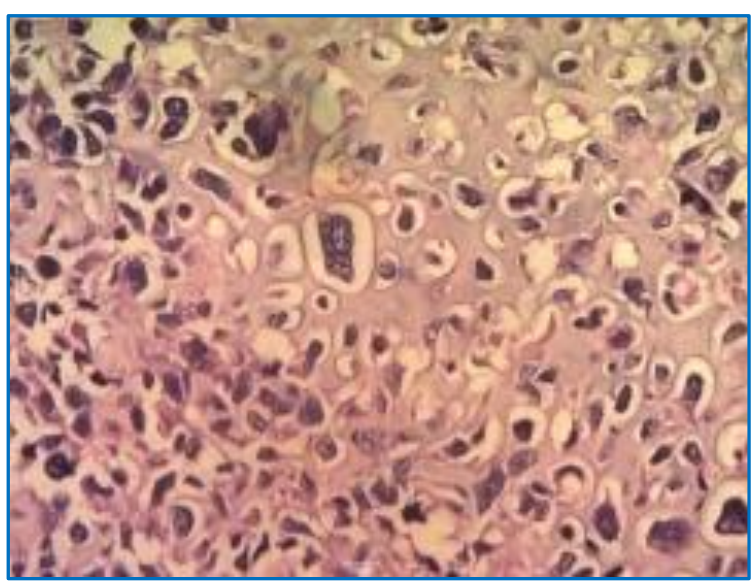

Fig. 5: Microphotograph showing chondrosarcomatous area with hypercellularity and marked nuclear atypia (H\&E 40X) 


\section{CASE REPORT}

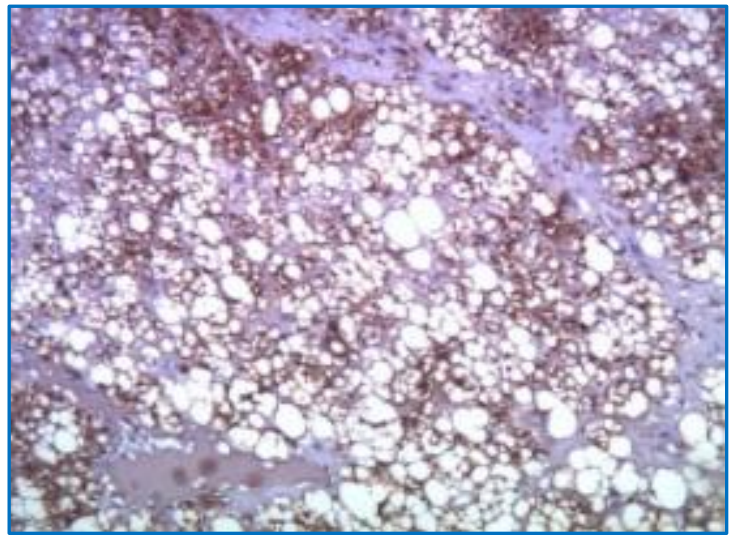

\section{Fig. 6: Microphotograph showing tumor cells positive for S100 on immunohistochemistry (10X)}

\section{AUTHORS: \\ 1. Tanu Agrawal \\ 2. Kanchan Garg \\ 3. Dupinder Kaur \\ 4. S. K. Sagar}

\section{PARTICULARS OF CONTRIBUTORS:}

1. Associate Professor, Department of Pathology, Shri Ram Murti Smarak Institute of Medical Sciences, Bareilly.

2. Junior Resident, Department of Pathology, Shri Ram Murti Smarak Institute of Medical Sciences, Bareilly.

3. Junior Resident, Department of Pathology, Shri Ram Murti Smarak Institute of Medical Sciences, Bareilly.

FINANCIAL OR OTHER COMPETING INTERESTS: None
4. Professor, Department of Surgery, Shri Ram Murti Smarak Institute of Medical Sciences, Bareilly.

\section{NAME ADDRESS EMAIL ID OF THE CORRESPONDING AUTHOR:}

Dr. Tanu Agrawal,

Department of Pathology,

Shri Ram Murti Smarak Institute of

Medical Sciences, Nainital Road,

Bhojipura, Bareilly-243202,

Uttar Pradesh, India.

E-mail: tanuagrawal510@yahoo.co.in

Date of Submission: 28/05/2015.

Date of Peer Review: 29/05/2015.

Date of Acceptance: 09/06/2015.

Date of Publishing: 15/06/2015. 